\title{
LA TRANSMISSION D'ARISTOTE PAR LES ARABES À LA CHRÉTIENTÉ OCCIDENTALE. UNE TROUVAILLE RELATIVE AU DE INTERPRETATIONE
}

\author{
Mr. Jean-Françoiș Monteil \\ Maître de conférence de Linguistique Générale à l'Université Michel de Montaigne Bordeaux III
}

A Daniel Guérineau

\begin{abstract}
RESUMEN
En el capítulo VII de Sobre la interpretación, Aristóteles mutila un sistema de tres pares de proposiciones contradictorias naturales. Aristóteles excluye el par de proposiciones universales naturales, los hombres son blancos, los hombres no son blancos, los cuales se contradicen mútuamente. Esta mutilación lleva consigo consecuencias indeseables. Los dos pares de proposiciones contradictorias naturales, consideradas exclusivamente por Aristoteles, Todos los hombres son blancos versus Algunos hombres no son blancos y Algunos hombres son blancos versus Ningun hombre es blanco son ilegitimamente identificadas con los dos pares de proposiciones contradictorias lógicas, las cuales constituyen el cuadrado lógico: A versus $\mathbf{O}$ y $\mathbf{I}$ versus $\mathbf{E}$ respectivamente. De este modo, se confunden el nivel del lenguaje natural y el de la lógica. Dicha mutilación aristotélica se ve disimulada por la traducción de las llamadas proposiciones indeterminadas. Para traducir estas últimas, semánticamente proposiciones particulares, los eruditos, excepto P.Gohlke utilizan las dos universales excluídas por el maestro! La obra de I. Pollak, publicada en Leipzig en 1913, nos revela el origen de este desliz de traducción casi universal: la versión árabe utiliza$\mathrm{da}$, desafortunadamente, por Al-Farabi en su comentario. Añadiendo los vértices $\mathbf{Y}$ y $\mathbf{U}$ a los del cuadrado, el hexágono lógico de Robert Blanché nos permite distinguir claramente la relación existente entre el sistema lógico y el sistema natural.
\end{abstract}

Palabras clave: Proposiciones indeterminadas (no cuantificadas) de Aristóteles, Proposiciones particulares lógicas-proposiciones particulares naturales, Cuadrado lógico, hexágono de Robert Blanché, Y; la tercera contraria, U la tercera subcontraria, Robert Blanché, Paul Gohlke, Isidor Pollak.

\footnotetext{
ABSTRACT

In chapter VII of On Interpretation, Aristotle alters a system of three pairs of natural contradictory propositions, in that he ignores the pair where two natural universals Men are white, Men are not white oppose each other contradictorily. This alteration has serious consequences: the two natural pairs, which Aristotle considers exclusively: All men are white versus Some men are not white and Some men are white versus No man is white are illegitimately identified to the two pairs of logical contradictories constituting the logical square: $\mathbf{A}$ versus $\mathbf{O}$ and $\mathbf{I}$ versus $\mathbf{E}$ respectively. Thus, the level of natural language and that of logic are confused. The unfortunate Aristotelian alteration is concealed by the translation of propositions known as indeterminates. To translate these, which, semantically, are particulars, all scholars, except for P.Gohlke, employ the two natural universals excluded by the master! The work of I. Pollak, published in Leipzig in 1913, reveals the origin of this nearly universal translation mistake: the Arabic version upon which Al-Farabi unfortunately bases his comment. In adding the vertices $Y$ and $U$ to the four ones of the square, the logical hexagon of Robert Blanche allows for the understanding of the manner in which the logical system and the natural system are linked.
} 
Key words: Indeterminate (or unquantified) propositions of Aristotle, logical particular propositions, natural particular propositions, logical particular propositions, natural particular propositions, logical particular propositions, natural particular propositions, Robert Blanché, Paul Gohlke, Isidor Pollak.

Par sa connaissance du grec et de l'arabe, l'auteur de cet article sait un peu comment s'est transmise la pensée d'Aristote, d'abord à l'orient islamisé puis à la chrétienté occidentale. L'article évoquera ce qu'Ibrahim Makdour appelle le «mouvement traducteur de l'Islam», mouvement patronné aux deuxième et troisième siècles de l'Hégire par les Califes de Bagdad. Il dira surtout pourquoi le rôle de la civilisation arabo-musúlmane dans la transmission d'Aristote peut intéresser la pensée moderne. Pour connaître la logique du maître dans sa force et dans ses limites, il n'est pas mauvais de connaître les traductions arabes du De Interpretatione et le commentaire d'Alfarabi. Peut-on négliger de regarder de ce côté quand on sait qu'Aristote pénétra en occident sous la forme de traductions latines de traductions arabes? Ce fait, que constate Albert Malet dans un manuel d'histoire de 1919, ne saurait laisser indifférent.

Etant à l'origine du carré logique, le chapitre VII du De Interpretatione est un texte fondateur, où chaque détail importe, où nulle imperfection ne peut être sans effet. Or, pratiquement tous les traducteurs rendent d'une manière erronée les propositions indéterminées d'Aristote. Il veut qu'elles soient des particulières signifiant Il y a des hommes blancs, Il y a des hommes non blancs, on les rend par des universelles: L'homme est blanc, L'homme n'est pas blanc. La cause prochaine de cette faute de traduction, aux conséquences insoupçonnées, est une version arabe, la cause première Aristote lui-même. En 1913, Isidor Pollak publie à Leipzig un ouvrage faisant état de deux traductions arabes du De Interpretatione. Le manuscrit de l'une est à Berlin, celui de l'autre à la Bibliothèque Nationale. Elles diffèrent dans leur manière de rendre les indéterminées. La traduction de Berlin respecte la lettre du texte grec, le texte arabe de Paris introduit la faute devenue quasi universelle. Seul, en effet, l'Allemand Gohlke fonde directement sa traduction sur le texte grec. Seul, il se conforme aux intentions d'Aristote et rend des particulières par des particulières.

\section{LE MOUVEMENT TRADUCTEUR DE L'ISLAM}

Dans les décennies qui suivirent la mort de Mahomet, la Perse sassanide et la majeure partie de la chrétienté orientale furent gagnées à l'Islam. Les fils du Prophète soumettaient à l'impôt chrétiens et juifs, qui avaient au sein de la communauté des croyants le statut de protégés, de dhimmis. Ils exigèrent aussi de leurs tributaires une autre contribution, intellectuelle celle-là. La jeune civilisation islamique puisa dans les trésors de la pensée antique. Evoquons ici le rôle de la Syrie car, depuis que Justinien avait fermé les écoles d'Athènes, la chrétienté de Syrie était le principal centre de la pensée hellénique. Les ouvrages grecs étaient traduits dans une langue sémitique proche de l'hébreu: le syriaque, forme tardive de l'araméen. Après la conquête musulmane, l'activité traductrice des chrétiens de Syrie s'amplifia. Quelques œuvres furent traduites du grec en arabe mais le plus souvent, on traduisait des textes grecs traduits en syriaque. Les Califes abbassides créèrent à Bagdad un atelier de traduction, appelé Bayt al Hikma Maison de la Sagesse. On attribue à Honayn Ibn Isaak et Isaak Ibn Honayn la traduction des livres d'Aristote qui nous intéressent. Ils savaient le grec et le syriaque, et avaient appris l'arabe. Pour acquérir à prix d'or les précieux manuscrits grecs, les Califes envoyaient des caravanes à Byzance. Ces textes étaient d'abord traduits en syriaque, puis du syriaque en arabe. Les traductions de l'Organon et de bien d'autres ouvrages gagnèrent l'Espagne sarrasine. A la couture du monde musulman et de la chrétienté, à Tolède mais aussi en Sicile, on traduisit activement de l'arabe en latin. De 1130 à 1150, l'Archevêque de Tolède, le Français Raymond d'Agen, employa des médiateurs juifs comme Yehuda Halevi et Aben Ezra, ou encore des 
savants chrétiens comme Gérard de Crémone. En plus de l'hébreu, les traducteurs juifs savaient l'arabe, le castillan, le latin. C'est ainsi qu'Aristote et les commentaires arabes pénétrèrent en occident et fécondèrent la chrétienté des Albert le Grand et des Saint Thomas d'Aquin.

\section{L'ORGANON D'ARISTOTE ET SON USAGE EN TERRE D'ISLAM}

On sait que l'Organon d'Aristote, c'est ce groupe de livres traitant de la logique, principalement du raisonnement et que le syllogisme déduit de propositions tenues pour vraies d'autres propositions ayant le même degré de vérité. Or le qiyas, le raisonnement, est la troisième source du fikh ou droit musulman, les trois autres étant le Coran, la tradition ou sunna, le consensus de la communauté croyante ou ijma. Le syllogisme aristotélicien est une logique à trois termes, puisqu'il est l'enchaînement, on le sait, de trois propositions. Il fut utilisé en Islam pour enrichir la démarche du qiyas. A l'origine, celui-ci représente une logique à deux termes puisqu'il s'agit primitivement d'un raisonnement fondé sur l'analogie. Le qiyas originel rapproche deux réalités semblables pour définir une règle de conduite relative à une réalité nouvelle. Quelle attitude doit-on, par exemple, adopter à l'égard du tabac, qui nous vient d'une Amérique découverte au quinzième siècle de l'ère chrétienne? Ceux qui en Islam en condamnent la consommation font valoir que celle-ci est assimilable à la consommation des boissons fortes et qu'en conséquence on ne peut prohiber la consommation de l'alcool sans combattre le taba-

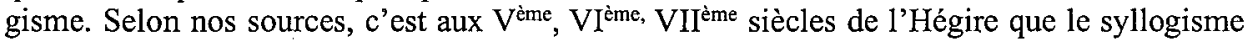
aristotélicien s'associa au qiyas primitif à deux termes. A partir de cette époque, disent les mêmes sources, la logique formelle d'Aristote fut enseignée dans les grandes mosquées.

\section{LES QUATRE PROPOSITIONS NATURELLES MARQUÉES DU GREC, À L'ORIGINE DU CARRÉ LOGIQUE}

Le syllogisme étant l'enchaînement de trois propositions, l'Organon contient un livre sur Ia proposition: le De Interpretatione. Le chapitre VII présente quatre propositions naturelles marquées, à l'origine du carré logique. L'inventeur de leur disposition en carré est Apulée de Madaure, philosophe latin du $2^{\text {ème }}$ après J-C, originaire de l'actuelle Tunisie. Ce logicien est aussi l'auteur de l'Âne d'or, l'un des meilleurs romans de la littérature latine. Voici ces propositions naturelles disposées en carré:

Tous les hommes sont blancs A

Pas anthropos leukos

Quelques hommes sont blancs I

Esti tis anthropos leukos
E Aucun homme n'est blanc Oudeis anthropos leukos

O Tous les hommes ne sont pas blancs Ou pas anthropos leukos

La linguistique appelle langue naturelle humaine une langue au sens usuel du terme. Pas anthropos leukos et Tous les hommes sont blancs sont des propositions naturelles parce que ce sont des phrases du grec et du français. Une langue est naturelle dans la mesure où elle est reçue en héritage et n'est pas fabriquée par la communauté qui l'utilise. On oppose les langues aux systèmes artificiels de signes comme le code de la signalisation routière ou les symboles de la logique algorithmique. La proposition logique $\mathrm{x}(\mathrm{x})$ et ses lectures concevables: Pour tout $x$, la fonction $f$ de $x$ est vérifiée ou encore Quel que soit le membre de l'ensemble les hommes, il est blanc sont des outils inventés par l'homme.

La phrase grecque sera ici suivie d'une traduction mot à mot, puis de la traduction adoptée. La phrase affirmative Pas anthropos leukos Tout homme (est) blanc Tous les hommes sont blancs et la négative Ou pas anthropos leukos Pas tout homme (est) blanc Tous les hommes ne sont pas blancs se contredisent mutuellement. De même, l'affirmative Esti tis anthropos leu- 
kos Il existe quelque homme blanc Quelques hommes sont blancs et la négative Oudeis anthropos leukos Pas un homme (est) blanc Aucun homme n'est blanc constituent un couple de contradictoires naturelles. Nous appelons contradictoires naturelles deux phrases assertives, l'une de forme dite affirmative, l'autre de forme dite négative, qui se contredisent mutuellement comme Il pleut et Il ne pleut pas. Chacune nie ce que l'autre affirme, affirme ce que l'autre nie. Il ne pleut pas affirme qu'il ne pleut pas et nie qu'il pleuve. Il pleut affirme qu'il pleut et, du même coup, nie qu'il ne pleuve pas. Il ne pleut pas est la contradictoire marquée, Il pleut la contradictoire non-marquée. L'habile doit parler comme le peuple, appelè négative la contradictoire marquée Il ne pleut pas, affirmative la contradictoire non-marquée $I l$ pleut. Mais il doit aussi avoir la pensée de derrière et se dire: Il ne pleut pas affirme qu'il ne pleut pas et $I l$ pleut nie qu'il ne pleuve pas. Dans la traduction detEsti tis anthropos leukos, le sujet est au pluriel, conformément à un usage observable dans l'article particulier du dictionnaire de Lalande. Ayant utilisé le pluriel pour rendre Esti tis anthropos leukos, nous en avons généralisé l'emploi. Dans Tous les hommes ne sont pas blancs, la négation, malgré sa place, porte sur le mot tous. La phrase a donc le sens de Quelques hommes ne sont pas blancs Quelques hommes sont non-blancs, elle n'a pas celui de Tous les hommes sont non-blancs, expression équivalant à Aucun homme n'est blanc.

A et I sont les deux premières voyelles de affirmo j'affirme. Traditionnellement, la proposition naturelle Tous les hommes sont blancs est identifiée à l'universelle logique affirmative A ou x $\mathrm{f}(\mathrm{x})$, la proposition naturelle Quelques hommes sont blancs à la particulière logique affirmative $\mathbf{I}$ ou $\exists \mathrm{x} f(\mathrm{x})$. $\mathbf{E}$ et $\mathbf{O}$ sont les deux voyelles de nego je nie. Trop souvent encore, la proposition naturelle Aucun homme n'est blanc est assimilée à l'universelle logique négative $\mathbf{E}$ ou $\mathrm{x} \sim \mathrm{f}(\mathrm{x})$, la proposition naturelle Tous les hommes ne sont pas blancs Quelques hommes ne sont pas blancs à la particulière logique négative $\mathrm{O}$ ou $\exists \mathrm{x} \sim \mathrm{f}(\mathrm{x})$. L'universelle logique affirmative $\mathbf{A}$ attribue la blancheur à toute l'humanité, la particulière logique affirmative $\mathbf{I}$ à au moins une partie de l'humanité. L'universelle logique négative $\mathbf{E}$ dénie la blancheur à toute l'humanité, la particulière logique négative $\mathbf{O}$ à au moins une partie de l'humanité. I particulière affirmative et $\mathbf{E}$ universelle négative sont des propositions logiques mutuellement contradictoires. La même relation de contradiction existe entre l'universelle logique affirmative $\mathbf{A}$ et la particulière logique négative $\mathbf{O}$. Les propositions naturelles d'Aristote sont à l'origine du carré logique, où nous les avons fait figurer conformément à la tradition. Pourtant, entre leur contenu et celui des propositions logiques qu'elles sont censées représenter la correspondance est, nous allons le voir, des plus approximatives.

\section{LES QUATRE PROPOSITIONS LOGIQUES À BIEN DISTINGUER DES NATURELLES PRÉCÉDENTES}

Voici un carré, où les quatre postes A I E $\mathbf{O}$ contiennent les propositions logiques:

\section{$\mathbf{A}$}

$x f(x)$

Quel que soit le membre

de l'ensemble les hommes, il est blanc

I

$\exists x f(x)$

Il existe au moins un membre

de l'ensemble les hommes qui est blanc
E

$\mathrm{x} \sim \mathrm{f}(\mathrm{x})$

Quel que soit le membre

de l'ensemble les hommes, il n'est pas blanc

O

$\exists \mathrm{x} \sim \mathrm{f}(\mathrm{x})$

Il existe au moins un membre

de l'ensemble les hommes qui n'est pas blanc

Ce qui prouve que les propositions naturelles d'Aristote correspondent imparfaitement aux quatre propositions logiques A I E O, c'est le fait que la logique a été contrainte de forger des expressions artificielles pour représenter avec exactitude le contenu de ses propositions à elle. 
Dans la logique moderne, I devient $\exists \mathrm{x} f(\mathrm{x})$ Il existe au moins un $x$ pour lequel la fonction $f$ de $x$ est vérifiée, $\mathrm{E}$ devient $\mathrm{x} \sim \mathrm{f}(\mathrm{x})$ Pour tout $x$ la fonction $f$ de $x$ n'est pas vérifiée. Pour que le lecteur perçoive mieux la différence entre les propositions logiques et les naturelles d'Aristote, nous coulons dans ces moules logiques la matière des propositions naturelles du chapitre VII: le sujet les hommes et l'attribut blanc. I se lit ici Il existe au moins un membre de l'ensemble les hommes qui est blanc, $\mathbf{E}$ se lit Quel que soit le membre de l'ensemble les hommes, il n'est pas blanc. Ces lectures ont pour vertu d'éviter l'identification d'une proposition naturelle à la proposition logique qui lui ressemble mais n'a pas exactement le même sens. Par exemple, 1'universelle affirmative logique $\mathbf{A}$ désigne le référent commun à deux universelles affirmatives naturelles différentes Tous les hommes sont blancs et Les hommes sont blancs. En conséquence, elle ne doit être confondue ni avec l'une ni avec l'autre. Un deuxième exemple: la proposition logique $\mathbf{I}$ Il existe au moins un membre de l'ensemble les hommes qui est blanc contient moins d'information que la proposition naturelle, avec laquelle on la confond souvent: Quelques hommes sont blancs. La thèse centrale de cet article, c'est qu'Aristote a éliminé de sa visée le couple naturel Les hommes sont blancs versus les hommes ne sont pas blancs et que les deux couples naturels retenus, inexactement interprétés, ont été indûment confondus avec deux couples de contradictoires logiques. Par exemple, le couple naturel Quelques hommes sont blancs versus Aucun homme n'est blanc ne doit pas être confondu avec le couple logique I Au moins un membre de l'ensemble les hommes est blanc versus $E$ Quel que soit le membre de ensemble les hommes, il n'est pas blanc. Il y a un lien entre cette confusion et l'éviction des propositions naturelles Les hommes sont blancs et Les hommes ne sont pas blancs.

\section{LA MUTILATION PÁR ARISTOTE DU SYSTÈME NATUREL}

Ce qui signale les propositions naturelles du chapitre VII, à l'origine du carré, c'est le fait que leur caractère d'universelle ou de particulière est grammaticalement marqué. Le mot tous indique que Tous les hommes sont blancs est une universelle, le mot quelques que Quelques hommes sont blancs est une particulière. Si nous insistons sur le caractère marqué de ces propositions, c'est pour mettre en lumière le fait qu'Aristote élimine Les hommes sont blancs et Les hommes ne sont pas blancs, phrases non-marquées très utilisées. Quand l'article les est employé avec une valeur généralisante, la phrase Les hommes sont blancs est, du point de vue logique, une universelle affirmative tout comme Tous les hommes sont blancs. Mais cette phrase est non-marquée car elle n'utilise pas de mot comme tous pour exprimer l'universalité. De même, les propositions Aucun homme n'est blanc et Les hommes ne sont pas blancs sont, du point de vue logique, des universelles négatives mais la première est une phrase marquée, la seconde une phrase non-marquée. Les phrases non-marquées Les hommes sont blancs et Les hommes ne sont pas blancs sont les grandes absentes de l'exposé aristotélicien, nous n'insisterons jamais assez sur ce fait. Aussi parlons-nous de mutilation par Aristote d'un système naturel. Cette mutilation a des conséquences graves: elle empêche l'esprit humain d'avoir une intelligence parfaite des mutuelles relations de la logique et de la grammaire. Les deux phrases non-marquées ne font pas double emploi avec les phrases marquées, dont elles partagent les valeurs logiques. Par exemple, si Tous les hommes sont blancs et Les hommes sont blancs sont toutes deux des universelles affirmatives, elles ont pourtant des sens différents. En effet toute phrase informant sur la réalité contredit une autre proposition. Partant, le sens d'une telle phrase, c'est ce qu'elle fait connaître de la réalité, certes, mais c'est aussi ce qu'elle est en tant que contradiction. Les hommes sont blancs contredit Les hommes ne sont pas blancs, une négative universelle tandis que Tous les hommes sont blancs contredit Quelques hommes ne sont pas blancs, une négative particulière. Les deux propositions sont des uni- 
verselles affirmatives, mais ne servant pas à contredire la même chose, elles n'ont pas la même signification.

\section{LES EMPOISONNANTES INDÉTERMINÉES DU TEXTE ARISTOTÉLICIEN}

Evinçant Les hommes sont blancs et Les hommes ne sont pas blancs, (ou ce qui revient au même ici L'homme est blanc et L'homme n'est pas blanc), Aristote mutile un système de six propositions. Hélas, cette mutilation est dissimulée par la présence dans le chapitre VII de deux propositions non-marquées dites indéterminées. Or, ces propositions n'ont rien à voir avec les propositions dont le grammairien déplore l'absence dans le De Interpretatione. Voici la forme de ces propositions indéterminées: Il y a blanc homme Esti leukos anthropos, Il n'y a pas blanc homme Ouk esti leukos anthropos. Le sens de l'affirmative est évident. Equivalant à la particulière affirmative marquée I Quelques hommes sont blancs, elle n'ajoute rien au carré. L'indéterminée négative $I l$ n'y a pas homme blanc, elle aussi, n'ajoute rien au groupe des phrases marquées. Mais elle a un défaut que n'a pas l'affirmative: elle pose un problème d'interprétation. Avec l'érudit allemand Gohlke, avec le savant espagnol Sanmartin, nous pensons que cette phrase a le sens de l'universelle négativé $\mathbf{E}$ Aucun homme n'est blanc. Or, à notre grande surprise, Aristote dit que cette proposition est comme Il y a homme blanc une proposition vraie. C'est nous dire implicitement qu'il faut interpréter Il n'y a pas homme blanc comme si on avait Il y a homme non-blanc et donc que l'indéterminée négative a le sens de la particulière négative marquée $\mathbf{O}$.

Cela dit, le débat sur l'interprétation à donner du Il n'y a pas homme blanc ne doit pas faire oublier l'essentiel: les indéterminées du chapitre VII n'ajoutent rien aux quatre propositions marquées à l'origine du carré et en négligeant les propositions Les hommes sont blancs et Les hommes ne sont pas blancs, Aristote mutile un système naturel de six phrases. Les propositions non-marquées éliminées expriment des significations spécifiques ne se retrouvant dans aucune des quatre propositions marquées retenues. Par exemple, bien qu'elles fassent connaître le même fait, les propositions Les hommes ne sont pas blancs et Aucun homme n'est blanc n'ont pas le même sens. En effet, elles ne contredisent pas la même chose. Donc si le fait majeur, c'est l'élimination par Aristote du couple Les hommes sont blancs versus Les hommes ne sont pas blancs, la difficulté présentée par l'indéterminée négative est un fait secondaire. Certes, Aristote veut qu'elle soit interprétée comme une particulière négative alors que, selon les érudits Gohlke et Sanmartin, il s'agit d'une universelle négative. Mais que l'indéterminée en question ait la valeur de $\mathbf{O}$ ou de $\mathbf{E}$, elle est, en tout état de cause, la redite inutile d'une proposition marquée du carré.

\section{LES CONSÉQUENCES FÂCHEUSES DE LA MUTILATION PAR ARISTOTE DU SYSTÈME NATUREL}

La partie 5 de l'article met en lumière le fait capital du chapitre VII: Aristote ignore le couple où Les hommes sont blancs, I'une des deux universelles affirmatives naturelles est opposée à Les hommes ne sont pas blancs, l'une des deux universelles négatives naturelles. Dans le tableau qui suit, ce sera le couple a. Sur les trois couples que comporte le système naturel, Aristote n'en étudie que deux: le couple où l'universelle affirmative marquée naturelle est opposée à la particulière négative naturelle, le couple où la particulière affirmative naturelle est opposée à l'universelle négative naturelle marquée. Dans le tableau, ces couples seront respectivement le couple b et le couple $\mathbf{c}$. Selon nous, les trois couples composent un système, que tronqua Aristote. 
a

Les hommes sont blancs

Les hommes ne sont pas blancs

b

Tous les hommes sont blancs

Quelques hommes ne sont pas blancs c

Quelques hommes sont blancs Aucun homme n'est blanc

Lélimination par Aristote du couple $\mathbf{a}$ a des effets fâcheux, que décrit notre article publié à Damas en 1996. Ici, ce sujet sera effleuré. En premier lieu, les universelles naturelles marquées retenues sont indûment identifiées aux universelles logiques $\mathbf{A}$ et $\mathbf{E}$. Ainsi, l'universelle affirmative logique $\mathbf{A}$ est confondue avec l'universelle affirmative naturelle du couple $\mathbf{b}$. Or, l'universelle affirmative logique $A$ se réalise non pas dans une mais dans deux universelles affirmatives naturelles, différant entre elles et par la forme et par le sens. Elle se réalise dans Tous les hommes sont blancs, certes, mais aussi dans Les hommes sont blancs du couple a. De même, l'universelle négative logique $\mathbf{E}$ se réalise dans deux universelles négatives naturelles différentes. Dans Aucun homme n'est blanc, mais aussi dans Les hommes ne sont pas blancs du couple a. En conséquence, il n'est pas légitime d'identifier l'universelle logique $\mathbf{E}$ à la seule universelle naturelle du couple $\mathbf{c}$. En second lieu; il y a une confusion des particulières naturelles avec les particulières logiques $\mathbf{I}$ et $\mathbf{O}$. Cette confusion est encore plus inadmissible que l'identification des universelles logiques aux universelles naturelles marquées. Comparons, par exemple, la particulière affirmative logique I et la particulière naturelle. Rappelons que pour le logicien, la particulière logique I consiste à dire Au moins un homme est blanc. Elle exclut donc le contenu des universelles naturelles négatives Aucun homme n'est blanc, Les hommes ne sont pas blancs et seulement ce contenu. Certes, elle n'exprime pas le contenu des universelles affirmatives naturelles Tous les hommes sont blancs, Les hommes sont blancs mais elle ne l'exclut pas non plus. Que ce contenu soit une possibilité est indiqué par l'expression au moins un. Bien différente, pour la quantité d'information, est la particulière affirmative naturelle Quelques hommes sont blancs. Comme la particulière affirmative logique I, elle exclut le contenu des propositions naturelles Aucun homme n'est blanc, Les hommes ne sont pas blancs mais elle exclut aussi celui des propositions Tous les hommes sont blancs, Les hommes sont blancs. Confondre la particulière affirmative logique $\mathbf{I}$ et la particulière affirmative naturelle est plus qu'une erreur, c'est une faute. Depuis plus de vingt siècles, c'est le secret de Polichinelle chez les doctes que la particulière affirmative naturelle Quelques hommes sont blancs contient plus d'information que la particulière logique I Au moins un homme est blanc.

L'identification des propositions logiques aux propositions naturelles est donc illégitime. L'universelle logique $\mathbf{A}$ se réalise dans la naturelle Pas anthropos leukos mais aussi dans l'autre universelle naturelle: Ho anthropos esti leukos L'homme est blanc. Ce Ho anthropos esti leukos est cette proposition dont Aristote n'a pas parlé. Cette phrase, si fầcheusement absente dans le chapitre VII, se distingue nettement de l'indéterminée qui s'y trouve: Esti leukos anthropos. Dans celle-ci, le esti est placé en tête et, pour cette raison, n'est pas enclitique mais accentué. D'autre part, il est associé à un nom sans article. Pour son interprétation, ces deux faits doivent être considérés conjointement. Ils font qu'il n'a probablement pas un rôle de copule et qu'il induirait plutôt, semble-t-il, le sens de il existe, il y a. C'est l'opinion de Gohlke, l'helléniste qui a examiné avec le plus de soin les indéterminées d'Aristote. Il traduit Esti leukos anthropos par Es gibt einen weissen Menschen Il y a un homme blanc. C'est également l'opinion implicite de l'érudit espagnol Sanmartin, si l'on considère la traduction qu'il donne spontanément de Ouk esti leukos anthropos, pendant négatif de Esti leukos anthropos. Spontanément, il traduit l'indéterminée négative par l'équivalent castillan du tour français Il n'y a pas homme blanc. Or, cela suppose que l'indéterminée affirmative est à interpréter comme Il y a homme blanc. Le contexte, en tout état de cause, et probablement aussi le donné syntaxique incitent à traduire l'indéterminée affirmative du chapitre VII par Il existe des hommes blancs. L'indéter- 
minée affirmative d'Aristote induit donc bien le sens de la proposition particulière marquée Esti tis anthropos leukos Il existe quelque homme blanc Quelques hommes sont blancs. Toutefois, ne cachons pas au lecteur que la présence d'un esti copule en 'tête de phrase, est, certes, rare, mais n'est pas une impossibilité.

Quoi qu'il en soit, dans Ho anthropos esti leukos, le nom est accompagné de l'article défini ho et esti est clairement une copule. Comme en français, l'article défini peut avoir en grec une valeur générique et la phrase Ho anthropos esti leukos est une des deux universelles affirmatives du système naturel. En grec comme en français, il y a donc deux universelles affirmatives naturelles différentes et il était illégitime d'identifier l'universelle logique $\mathbf{A}$ à la seule naturelle Pas anthropos leukos. La proposition logique $\mathbf{A}$ ne doit plus être identifiée à Pas anthropos leukos car elle exprime le contenu référentiel commun.à Pas anthropos leukos et à Ho anthropos esti leukos. Ces propositions naturelles de forme différente ont des sens différents, même si elles font connaître le même fait.

\section{LES TRADUCTIONS DISSIMULENT LA MUTILATION ARISTOTÉLICIENNE}

La quasi totalité des traductions occulte le fait qu'Aristote élimine le couple a. Avec insistance, nous en déplorons l'absence dans le chapitre VII. Or, à l'exception de Gohlke, tous les traducteurs utilisent les deux universelles de ce couple pour rendre les indéterminées d'Aristote, qui sont à interpréter comme des particulières. Comment expliquer cette aberrante traduction pratiquement universelle? Voici une explication. Désorientés par l'incroyable Ouk esti leukos anthropos, les premiers traducteurs ne savaient pas comment rendre les indéterminées. Hélas, ils furent tentés d'employer comme phrases traductrices ces universelles du couple a, qui, ayant été évincées du système naturel par Aristote, étaient en situation d'électrons libres. Et c'est ainsi que des indéterminées, qui sont des particulières, se trouvent traduites par des universelles. Pour un Français, la voie d'accès au De Interpretatione, c'est la traduction de J.Tricot éditée chez Vrin. Les indéterminées y sont traduites par L'homme est blanc et L'homme n'est pas blanc.

Si l'enjeu était simplement une traduction fidèle des indéterminées du chapitre VII, la faute serait peu grave. Ces propositions sont destinées à ne jamais être utilisées par le logicien aristotélicien dans la pratique de son art. Pourquoi le seraient-elles? Les indéterminées d'Aristote, on l'a vu, sont des redites de propositions marquées du carré et ce, quelle que soit l'interprétation que l'on donne de la négative Ouk esti leukos anthropos. Comme nous comprenons le philosophe, à qui il importe peu que ces inutilités soient bien ou mal traduites! Seulement voilà, la traduction traditionnelle présente l'inconvénient de dissimuler à plaisir le fait majeur, et si lourd de conséquences: l'éviction par Aristote du couple L'homme est blanc versus L'homme n'est pas blanc. Comment les gens pourraient-ils s'aviser que le maître élimine le couple a et mutile ainsi le système naturel, quand ils voient ce même couple a constamment utilisé pour traduire les indéterminées?

\section{GRANDEUR DE GOHLKE. ISIDOR POLLAK ET LES DEUX TRADUCTIONS ARABES}

Les indéterminées d'Aristote empoisonnent la tradition philosophique depuis plus de deux mille ans. Exprimant le sentiment général des savants attentifs et, partant, décontenancés, l'Anglais Ackrill écrit: «It is a pity that Aristotle introduces indefinite statements at all». Aristote ne nous demande-t-il pas d'interpréter une universelle fausse $I l n$ 'y a pas homme blanc $O u k$ esti leukos anthropos comme une particulière vraie $I l y$ a des hommes non-blancs, autrement 
dit, comme si l'on avait en grec Esti ou leukos anthropos avec la négation ou, ouk portant non pas sur esti il y a mais sur leukos blanc?

Comment alors traduire les indéterminées du chapitre VIÍ? L'attitude de Gohlke est exemplaire, faite qu'elle est de lucidité et de respect des intentions d'Aristote. Puisque le maître veut que ses indéterminées soient des particulières, il les traduit par des particulières. Discipliné, il traduit donc Esti leukos anthropos par Il y a des hommes blancs et Ouk esti leukos anthropos par Il y a des hommes non-blancs. Mais, lucide, il signale en note que l'indéterminée négative signifie en réalité Aucun homme n'est blanc. Ceux qui pourraient trouver inconvenante cette attitude critique de Gohlke doivent savoir qu'Aristote lui-même reconnaît que son indéterminée négative a l'air de signifier Aucun homme n'est blanc. Dès 1936, Gohlke propose dans son ouvrage Die Enstehung der Aristotelischen Logik une traduction du chapitre VII. Elle est accompagnée d'un commentaire, où il exprime ses vues originales et justes sur les indéterminées. Après la seconde guerre mondiale, il traduit toute l'œuvre d'Aristote pour la maison Schöningh de Paderborn. En 1951 paraît sa traduction du De Interpretatione avec la précieuse note 10. Nous avons traduit cette note dans un article intitulé Une exception allemande, que la Revue des Etudes Anciennes a publié en 2001. A notre connaissance, Gohlke est le seul à traduire les indéterminées du chapitre VII par des particulières. Voici, par exemple, les trois traductions qu'il propose de l'indéterminée négative: Il arrive qu'homme ne soit pas blanc Quelquefois homme n'est pas blanc Il y a un homme non blanc. Ces trois phrases sont des particulières rendant une proposition qu'Aristote nous enjoint d'interpréter comme une particulière. On notera avec intérêt que Gohlke, le premier à suggérer que l'indéterminée négative a le sens d'une universelle négative, est aussi le seul à traduire l'indéterminée négative conformément aux intentions d'Aristote.

Ce qu'il faut bien appeler un fait déroutant jeta dans le plus grand désarroi les traducteurs cherchant à rendre en arabe les indéterminées du texte grec. Dans un ouvrage publié en 1913 à Leipzig, Isidor Pollak nous apprend l'existence de deux traductions arabes du De Interpretatione. Il nous permet de constater que ces traductions ne rendent pas de la même manière les indéterminées d'Aristote. Le manuscrit de la première se trouve à Berlin dans un ensemble appelé Codex syriaque. Le donné formel du texte grec y est rendu avec une exacte fidélité. L'indéterminée affirmative Esti leukos anthropos y est rendue par Yujadu insanun abyadan Il y a un homme blanc, la négative Ouk esti leukos anthropos par Laysa abyadan insanun Il n'y a pas de blanc homme. Le manuscrit de la seconde se trouve à la Bibliothèque Nationale. Les indéterminées y sont traduites par Al insan abyad L'homme est blanc et Al insan laysa abyad L'homme n'est pas blanc. Encore une fois, la traduction est fautive car elle rend par des universelles fausses les indéterminées d'Aristote, qui sont à interpréter comme des particulières vraies. Encore une fois, elle introduit dans les indéterminées d'Aristote un article défini générique et fait du verbe esti une copule. Avec l'article al générique, Al insan abyad fait connaître le même fait que Kull insan abyad Tout homme est blanc. Cette faute de traduction s'est invétérée dans la tradition aristotélicienne, elle est à l'origine de bien des commentaires embarrassés, poussifs, viciés à la racine. Elle s'observe dans toutes les traductions ultérieures, celle de Gohlke étant l'heureuse exception. Certes, dans les deux versions latines, dont disposait Saint Thomas d'Aquin, la lettre des indéterminées d'Aristote est excellemment reproduite par Est albus homo, Non est albus homo. Mais ces traductions latines de Esti leukos anthropos et de Ouk esti leukos anthropos ne sont pas celles qui importent car elles n'ont eu aucune influence. En revanche, les phrases latines qu'il faut considérer, en raison de leurs effets dans la tradition, ce sont Homo est albus et Homo non est albus que le doctor angelicus substitue aux précédentes dans son commentaire sur le De Interpretatione. Or, comme le montre notre article sur Gohlke, Homo est albus, par exemple, équivaut très probablement non à Il y a homme blanc mais à L'homme est blanc.

Grâce à Isidor Pollak, il est possible de reconstituer le fatal scénario. Une première traduction arabe rendit les indéterminées en respectant la forme qu'elles ont en grec. Elles furent 
traduites par des phrases signifiant $I l y$ a des hommes blancs et Il n'y a pas d'homme blanc. On s'aperçut alors que la seconde ne peut avoir le sens d'une particulière négative. Il aurait fallu constater la difficulté et, comme Gohlke, substituer un $I l y$ a des hommes non blancs à Il n'y a pas d'homme blanc. Au lieu de cela, on remodela les indéterminées d'Aristote, en y introduisant un article défini et en faisant de esti une copule. Cela n'arrangea rien, le L'homme n'est pas blanc avec article générique, étant une universelle négative tout comme $I l n^{\prime} y$ a pas d'homme blanc. Les choses même s'aggravèrent, l'indéterminée affirmative devenant, elle aussi, une universelle fausse sous la forme de L'homme est blanc.

\section{F.W ZIMMERMANN ET SA TRADUCTION D'ALFARABI}

La traduction de Berlin respecte la lettre du texte grec, celle de Paris la malmène. Malheureusement, c'est la seconde qui a fait l'objet du commentaire d'Alfarabi. D'un Alfarabi, visiblement embarrassé par les phrases $A$ l insan abyad, Al insan laysa abyad. A juste titre, elles lui paraissent avoir le contenu de Tous les hommes sont blancs, Aucun homme n'est blanc respectivement. Mais abusé par la traduction fautive, il pense à tort que les indéterminées d'Aristote comportent un article défini et utilisent est $i$ comme copule. Il pense à tort que les tours grecs équivalant aux phrases L'homme est blanc et L'homme n'est pas blanc du français signifient parfois Certains hommes sont blancs et Certains hommes ne sont pas blancs respectivement. En 1981, F.W Zimmermann publie à 0xford sa traduction anglaise du commentaire d'Al-Farabi sur le De Interpretatione. Ce texte fait apparaître clairement qu'Al-Farabi est induit en erreur par la traduction du manuscrit de Paris, traduction attribuée à Ibn Hunayn. Ne croit-il pas de bonne foi que les indéterminées dans le texte grec comporte l'arthron, l'article défini? Citons Al-Farabi: «The subject in opposite unquantified sentences is expressed by a noun with the definite article, which is something common to every language. For the way to express an unquantified subject in Persian is to attach to its name a particle corresponding to the article al in Arabic. Similarly in Greek. The Greek particle which corresponds to the Arabic al is known among Greeks grammarians as arthron)?.

Son commentaire sur les indéterminées du chapitre VII eût été moins embarrassé, s'il avait eu une connaissance directe du texte grec et rien ne montre davantage son intelligence que cet embarras même. Un autre passage que nous allons citer le prouve. En substance, il dit en effet que $A$ linsan abyad L'homme est blanc avec al générique a naturellement le sens d'une universelle et fait connaître le même fait que l'universelle marquée Kull insan abyad Tout homme est blanc (kull a le sens du mot français tout). Mais d'un autre côté, il se voit contraint d'imaginer un autre usage de cette phrase. Voici ce qu'il écrit au sujet de l'article défini grec ho: «In connexion with the subject of an unquantified statement, it signifies one of the two things first mentionned. If it is used to signify that the notion is plain and not delimited by a quantification, the two sentences are not contrary. But if it is used in the meaning of every, then the sentences are indeed contrary. For whenever we say (the) man in the sense of every man, (the) man is white means every man is white and (the) man is not white means no man is white». Avant de commenter, disons un mot de ce the entre parenthèses. Il est le fait de l'anglophone Zimmermann. Celui-ci donne à la phrase Man is white la forme qu'elle aurait, si les noms man et woman ne constituaient pas l'exception bien connue des anglicistes. L'anglais dit The dog is a faithful animal mais Man says woman is frivolous. Dans ce texte, Al-Farabi attribue deux usages aux phrases L'homme est blanc, L'homme n'est pas blanc. Dans le premier, les deux propositions ne sont pas contraires, elles peuvent être vraies ensemble, s'énoncer l'une et l'autre dans le même discours. Si nous admettons un instant que L'homme est blanc puisse traduire une indéterminée d'Aristote signifiant Certains hommes sont blancs et que du même coup L'homme $n$ 'est pas blanc puisse signifier Certains hommes ne sont pas blancs, alors, c'est vrai, on pourra dire à la fois que l'homme est blanc et que l'homme n'est pas blanc. Sur ce beau modè- 
le, on pourra dire à la fois que l'homme joue de la trompette et qu'il ne joue pas de la trompette, que le Français aime la vodka et qu'il n'aime pas la vodka etc. Dans le second usage, qui est celui des souks de Damas et de Tunis, L'homme est blanc avec article générique veut dire que tous les hommes sont blancs et L'homme n'est pas blanc qu'aucun ne l'est. En réalité, il n'est pour les deux phrases qu'un seul usage: le second. Le premier est imaginaire, né qu'il est d'une faute de traduction gigantesque.

\section{LUCIDITÉ DE PORT-ROYAL}

En parlant de Pollak et de Zimmermann, nous avons mené le lecteur dans la galerie des ancêtres. Achevons la visite en évoquant Port-Royal. Dans leur Logique, Nicole et le grand Arnaud circonscrivent l'abcès avec leur acuité d'esprit coutumière. L'Observation VI du chapitre XIII compare deux indéterminées: L'homme est raisonnable, L'homme est juste. Pour les scolastiques, la première est une universelle, la matière du jugement y étant nécessaire alors que la seconde est une particulière, la matière du jugement y étant contingente. Pour Arnaud et Nicole, grammairiens très sûrs, il en va tout autrement. Les deux sont des universelles car dans les deux, la forme est identique et comporte l'article générique. La première est une universelle vraie, l'homme étant par définition l'animal mortel doté de la raison tandis que la seconde est une universelle fausse, la nature humaine ne s'associant à la justice que par accident. Pour parler comme Gohlke, lecteur attentif de Porphyre et traducteur rigoureux du chapitre VII, il arrive qu'homme soit juste, es kommt vor, dass Mensch gerecht ist.

Le lecteur l'aura compris. S'est instauré l'usage de distinguer deux indéterminées: une indéterminée universelle, une indéterminée particulière. En terre d'Islam et dans la chrétienté, un exposé scolastique présente les propositions marquées dont use le syllogisme, puis il dit un mot des indéterminées. Pour s'en débarrasser, il les réduit en les ramenant à des propositions marquées. J.Tricot, le traducteur français du De Interpretatione, est l'auteur d'un Traité de logique formelle, publié chez Vrin. Dans le chapitre 4 de cet ouvrage se trouve un paragraphe au titre révélateur Réduction des indéterminées. Il écrit: «La proposition indéterminée ne constitue pas une classe à part. Elle est ou universelle ou particulière selon l'intention de celui qui l'énonce. Par exemple, si je dis l'homme est mortel en donnant au sujet la signification générale de créature raisonnable, c'est comme si je disais Tout homme...». En bon disciple d'Alfarabi, Moshé Maïmonide donne deux exemples d'indéterminée: L' homme est un animal, qui répond à l'universelle marquée Tout homme est un animal et L'homme écrit qui fait écho à la particulière marquée Quelques hommes écrivent.

Ce que critiquent Arnaud et Nicole dans l'Observation VI; c'est le discours thomiste selon lequel L'homme est un animal est une universelle parce que la qualité d'être vivant s'associe nécessairement à l'humanité tandis que L'homme est blanc est une particulière parce que la blancheur s'y associe d'une façon contingente. Le texte de Saint Thomas d'Aquin auquel ils songent se trouve dans les In Aristotelis stagiritae nonnullos libros commentaria, plus précisément dans les Lectiones X,XI du commentaire sur le De Interpretatione. Voici leur formulation de cette thèse adverse sur l'indéterminée (qu'ils appellent indéfinie): «La plupart des philosophes disent qu'elle doit passer pour une universelle dans une matière nécessaire et pour particulière dans une matière contingente». Voici comment ils rejettent cette thèse: «La proposition indéfinie doit passer pour universelle en quelque matière que ce soit. Et ainsi dans une matière contingente, elle ne doit pas être considérée comme une proposition particulière mais comme une universelle qui est fausse». Nous faisons nôtre, évidemment, cette opinion. Pour le grammairien, L'homme est un animal est une universelle et une universelle vraie parce qu'il est constant que tout homme est un être vivant. La phrase L'homme est blanc est également une universelle mais une universelle fausse parce qu'il est constant que certains hommes seulement sont blancs de peau. Nous notons sans surprise que dans son paragraphe Réduction des indé- 
terminées, Tricot donne bien un exemple d'indéterminée à valeur d'universelle mais ne donne aucun exemple d'indéterminée à valeur de particulière. Il se garde de donner comme exemple ce L'homme est blanc, par lequel il traduit l'indéterminée Esti leukos anthropos d'Aristote. Pour lui aussi, ce L'homme est blanc est une universelle.

\section{HUIT PROPOSITIONS SUR LE CHAPITRE VII DU DE INTERPRETATIONE}

1. Le fait majeur du chapitre VII, c'est le fait qu'Aristote y mutile un système naturel de trois couples de contradictoires naturelles. Il évince le couple de contradictoires naturelles Ho anthropos esti leukos versus Ho anthropos ouk esti leukos, autrement dit, le couple où deux universelles naturelles Les hommes sont blancs, Les hommes ne sont pas blancs s'opposent l'une à l'autre.

2. Les quatre propositions marquées du chapitre VII se groupent en deux couples de contradictoires naturelles: Pas anthropos leukos Tout homme est blanc versus $\mathrm{Ou}$ pas anthropos leukos Tout homme n'est pas blanc, Esti tis anthropos leukos Il y a quelques hommes blancs versus Oudeis anthropos leukos Pas un homme n'est blanc. Ces deux couples de contradictoires naturelles sont à l'origine du carré logique. Cependant, malgré ce lien de paternité, il n'est pas légitime de les identifier aux deux couples de contradictoires logiques constituant le carré des logiciens: $\mathbf{A}$ versus $\mathbf{O}$, I versus $\mathbf{E}$.

L'universelle affirmative $\mathbf{A}$ appréhende le référent commun à deux universelles naturelles: Tous les hommes sont blancs, Les hommes sont blancs. Elle ne doit donc pas être identifiée à la seule universelle naturelle marquée Tous les hommes sont blancs. L'universelle négative $\mathbf{E}$ appréhende le référent commun à deux universelles naturelles Aucun homme n'est blanc, Les hommes ne sont pas blancs. Elle ne doit donc pas être identifiée à la seule universelle marquée Aucun homme n'est blanc. Quant aux particulières logiques I et $\mathbf{O}$, elles ne doivent pas être identifiées aux particulières naturelles Quelques hommes sont blancs et Quelques hommes ne sont pas blancs (ou Tous les hommes ne sont pas blancs). A l'instar des universelles, les particulières naturelles sont saturées d'information et contiennent plus d'information que les particulières logiques.

3. Les indéterminées d'Aristote Esti leukos anthropos et Ouk esti leukos anthropos sont inutiles car elles n'ajoutent rien aux propositions marquées du chapitre VII. L'indéterminée affirmative Esti leukos anthropos Il y a blanc homme équivaut à la particulière marquée Esti tis anthropos leukos Il y a quelque homme blanc Quelques hommes sont blancs. Quelle que soit l'interprétation qu'on en donne, l'indéterminée négative Ouk esti leukos anthropos équivaut, elle aussi, à une proposition marquée du chapitre VII. Si, comme le veut Aristote, on lui donne le sens d'une négative particulière, autrement dit, si on l'interprète comme si l'on avait Esti ou leukos anthropos Il y a homme non blanc, elle équivaut à la négative particulière marquée Ou pas anthropos leukos Tout homme n'est pas blanc (ou Quelques hommes ne sont pas blancs). Si elle a le sens de Il n'y a pas d'homme blanc, comme cela est probable en raison de la place de la négation ou(k), elle a la valeur d'une négative universelle et équivaut à l'universelle marquée Oudeis anthropos leukos Aucun homme n'est blanc.

4. Les érudits Gohlke et Sanmartin voient dans l'indéterminée négative Ouk esti leukos anthropos une universelle, Aristote lui-même reconnait qu'elle a tout l'air d'être synonyme de Oudeis anthropos leukos. Néanmoins, puisque Aristote veut que son indéterminée négative ait le sens d'une particulière, le traducteur a le devoir strict de se conformer à cette volonté et de rendre les deux indéterminées comme des particuliè- 
res. Il doit imiter l'Allemand Gohlke, qui les traduit par Il y a des hommes blancs, II y a des hommes non blancs.

5. Ce Ouk esti leukos anthropos, qui a probablement le sens d'une universelle mais doit se traduire par une particulière, désorienta les premiers traducteurs d'Aristote. Pour rendre les indéterminées du chapitre VII, qui, selon Aristote, sont des particulières, ils utilisèrent les deux universelles évincées L'homme est blanc, L'homme n'est pas blanc. Cette faute de traduction s'est universellement répandue. Seul l'Allemand Gohlke en a été préservé.

6. Mauvaise en elle-même, cette traduction a, en oútre, pour effet d'occulter la mutilation par Aristote d'un système naturel. Pour traduire les inutiles indéterminées du chapitre VII, elle utilise les deux universelles naturelles éliminées par le maître.

7. A cause de cette mutilation aristotélicienne, les plans de la langue naturelle et de la logique sont confondus, deux couples de contradictoires naturelles sont indûment identifiés aux deux couples de contradictoires logiques constitutifs du carré.

8. Par ses deux postes: $\mathbf{Y}$ et $\mathbf{U}$ venant s'ajouter aux quatre postes du carré, l'hexagone logique de Robert Blanché rend leur intégrité au système logique et au système naturel, ces deux systèmes étant à la fois distincts et liés.

Les sept premières propositions sont une concentration des idées exprimées dans ce travail. Puisque la proposition 8 évoque Blanché, un mot de son hexagone logique. En 1966, ce logicien publie Structures intellectuelles, où il substitue l'hexagone au carré. Avec ses six postes, l'hexagone est une forme plus puissante car il comporte deux postes faisant fâcheusement défaut dans le carré. Il ajoute aux quatre postes $\mathbf{A}$ I $\mathbf{E} \mathbf{O}$ le poste $\mathbf{Y}$ représentant la quantité partielle et le poste $\mathbf{U}$ représentant l'exclusion de la quantité partielle. Ces deux additions sont appelées à avoir des effets importants dans les domaines de la logique et de la grammaire générale. Le poste $\mathbf{Y}$ représente le référent des particulières naturelles, qui contiennent plus d'information, on l'a vu, que les particulières logiques. Le poste $\mathbf{U}$ représente la valeur commune de ces deux contradictoires naturelles qu'Aristote n'a pas voulu considérer, mutilant ainsi un système naturel. Mais cela est la matière d'un autre article.

\section{BIBLIOGRAPHIE-NOTES-COMPLEMENTS}

\section{Alfarabi}

Al-Farabi's Commentary and short treaty on Aristotle's De Interpretatione, transl, intr, notes, F.W.Zimmermann, London, Oxford U.P, 1981, p. 62, 63.

Les deux citations d'Alfarabi de notre article viennent des pages 62 et 63 de la traduction anglaise. On lira avec profit page 63 la note 1 de Zimmermann sur ce passage du Commentaire où Al-Farabi attribue indûment un article défini aux indéterminées du texte grec d'Aristote: In the Greek text, however, the example sentences at 17b 9f are without article.

Apulée The Peri Hermeneias of Apuleius of Madaura, Lat.text, Eng. Transl D.Londey, C. Johanson, in The logic of Apuleius, Philosophia antiqua, volume XLVII, E.J.Brill, Leiden 1987. Partie III, p 83.

\section{Aristote}

- Aristoteles, Die Lehrschriften, édit,trad,com. de P.Gohlke, Bde.1.9.1947-1961. Bd. 2,1: Kategorien und Hermeneutik. Paderborn, 1951.

- Aristoteles, Tratados de Logica (Organon) II: Sobre la Interpretacion..., intr. trad. notes de C.Sanmartin, Editorial Gredos, Madrid,1988, p 45, note 53.

- Kitab Aristutalis bari arminyas, Ishaq b. Hunayn, éd. Badawi, Koweit, 1980.

- Aristotle's Categories and De Interpretatione, translated with notes by J.L Ackrill, Oxford University Press, 1963. 
J.L Ackrill traduit les indéterminées d'Aristote Esti leukos anthropos, Ouk esti leukos anthropos par A man is white, A man is not white alors que les autres traductions anglosaxonnes les rendent par Man is white, Man is not white. Saluons le scrupule que cela manifeste, mais il est permis de douter que l'effort ait abouti. En français, la phrase $U n$ homme est mortel est comme L'homme est mortel une proposition universelle du point de vue logique. Langlais diffère-t-il du français sur ce point? Nous conseillons au lecteur de consulter à ce sujet l'ouvrage de John Lyons: Semantics 1 Cambridge University Press 1977, Volume I, 7.2, Reference, p194.

- Organon II. De l'interprétation, J. Tricot, Vrin, 1959.

Arnaud (A) et Nicole (P) La logique ou l'art de penser, Flammarion, 1970, p. 198-204, chap. XIII de la seconde partie de la logique.

Badawi (A) La transmission de la philosophie grecque au monde arabe, J.Vrin, 1968, Etudes de philosophie médiévale.

\section{Blanché (R)}

- Structures intellectuelles, Vrin, 1966. Lire séance tenante les chapitres III et IV.

- La logique et son histoire, Armand Colin, Paris,1970. p.34

Brunschwig $(\mathbf{J})$ «La proposition particulière chez Aristote», dans Cahiers pour l'analyse, cité par Blanché dans La logique et son histoire, p. 34.

Notre article évoque un fait majeur: la différence qu'il y a entre les particulières logiques et les particulières naturelles. Comme le dit avec bonheur le Professeur Brunschwig, les particulières logiques contiennent moins d'information que les universelles logiques auxquelles elles sont contradictoirement opposées. Dans notre terminologie, nous disons que pour la quantité d'information, il y a une dissymétrie entre les universelles logiques et les particulières logiques: les universelles logiques sont saturées d'information alors que les particulières logiques ne le sont pas. En revanche, dans le système naturel, toutes les propositions sans exception sont saturées d'information. Les particulières naturelles contiennent autant d'information que les universelles naturelles. La particulière naturelle Certains élèves font de l'anglais s'oppose à Aucun élève ne fait de l'anglais, mais aussi à Tous les élèves font de l'anglais. Elle a pour référent ce que notre article de Damas appelle la quantité partielle et que symbolise le poste $\mathbf{Y}$ de l'hexagone de Blanché. Brunschwig écrit: "La particulière "logique"a eu quelque peine à tuer la particulière "naturelle" mais elle a fini par y arriver». Notre article de Damas est une tentative de ressusciter la particulière naturelle.

Gohlke (P) Die Entstehung der Aristotelischen Logik, Junker und Dünnhaupt Verlag, Berlin 1936. p. 27

Lalande (A) Vocabulaire technique et critique de la philosophie, Quadrige/Presses Universitaires de France, 1991. p. 744

Lambert (Johann-Heinrich) Neues Organon, Akademie-Verlag Berlin, 1990, Erster Band, Dianoiologie, p 74, paragraphe 143.

En logique, l'Alsacien est novateur. Comme la tradition indienne, comme le Blanché de Structures intellectuelles, il perçoit l'importance de la quantité partielle. Le carré d'Aristote représente la totalité et la quantité zéro dans ses postes $\mathrm{A}$ et $\mathrm{E}$ mais pas la quantité partielle. Il faudra attendre l'hexagone de Blanché pour que cette quantité ait une représentation explicite dans le poste $\mathrm{Y}$ de cet hexagone.

Lulle (Raymond) Die Neue Logik (Nova Logica), éd.crit. de Charles Lohr, texte lat., trad.all. de Vittorio Hösle et Walburga Büchel, Hambourg, Felix Meiner, 1985. p.188-189.

Nous notons que les quatre phrases latines, utilisées par Lulle pour remplir les quatre cases du carré logique sont les mêmes que les quatre phrases arabes utilisées par Maïmonide pour le même usage.

Lyons (J) Semantics 1 Cambridge University Press 1977, Volume I, 7.2, Reference, p194.9.7,Marked and unmarked terms, p 309. 
Certains se demanderont si oui ou non la traduction d'Ackrill $A$ man is white représente un progrès par rapport à la traduction de Cook Man is white. Nous leur conseillons de lire Lyons.

Maïmonide (M) Traité de logique. Traduction, présentation et notes de Rémi Brague, Coll. Midrash, Desclée de Brouwer, Paris, 1996.

Makdour (I) L'Organon d'Aristote dans le monde arabe, deuxième édition, Paris, 1969.

Malet (A) Histoire moderne (1498-1715), classe de seconde, Hachette, 1919, p152.

Monteil (J.F)

- «De la traduction en arabe et en français d'un texte d'Aristote: le chapitre VII du Peri Hermeneias», in Bulletin d'Etudes Orientales, Tome XLVIII, Année 1996. Institut français d'Etudes Arabes de Damas.

L'article de 96 montre l'importance des problèmes de traduction évoqués à propos de Gohlke. Inspirées par une relecture du chapitre VII à la lumière de Structures intellectuelles de Blanché, ces vingt pages sont une thèse sur la contradiction naturelle en tant qu'elle est à distinguer de la contradiction logique. Ce qui caractérise la contradiction naturelle, $c$ 'est le fait que les deux contradictoires naturelles sont l'une et l'autre saturées d'information. La contradiction logique, elle, oppose une proposition saturée d'information à une proposition contenant moins d'information.

- «Une exception allemande: la traduction du De Interpretatione par le Professeur Gohlke. La note10 sur les indéterminées d'Aristote). (paru dans la Revue des Etudes Anciennes en Décembre 2001)

- «De la traduction en hébreu d'un texte arabe de Maïmonide: le chapitre II du Maqala fi sina at al mantiq ou Traité de logique», (paru en français dans les Cahiers de Tunisie en 2001)

L'article se fonde sur le Peri hermeneias d'Apulée pour expliquer les propos de Maïmonide sur la proposition indéterminée. Il explique du même coup la différence entre le texte protocanonique arabe et les trois versions hébraïques de ce texte majeur.

Monteil (V) Clefs pour la pensée arabe, Séghers.

Pollak (I) «Die Hermeneutik des Aristoteles in der arabischen Uebersetzung des Ishak ibn Honain», (Abhandlungen für die Kunde des Morgenlandes, XIII, Band, numéro 1), Leipzig, 1913, p. 11, notes 189 et 190.

Porphyre Isagoge, traduction et notes de J.Tricot, Vrin, Paris, 1947.

Saint Thomas d'Aquin In Aristotelis stagiritae nonnullos libros commentaria, Paris, 1889, L.Vivès, Tome XXII, livre I, lectiones X, XI, p 30-38.

Il est probable que le tour Est albus homo (ou Est homo albus) des deux traductions latines, l'antiqua et la recens, équivaut à Esti leukos anthropos Il existe homme blanc. En revanche, le tour Homo est albus, utilisé par Saint Thomas dans son commentaire sur le De Interpretatione, a probablement le sens de L'homme est blanc Ho anthropos esti leukos (voir J.F Monteil «Une exception allemande...» p 422).

Tricot (J) Traité de logique formelle, Vrin, 1976, chapitre quatrième, p 112 et p 113.

Jean François Monteil

c. e. Jean-Francois.Monteil@u-bordeaux3.fr 